\title{
DONNÉES RÉCENTES SUR LES CARACTÉRISTIQUES MORPHOLOGIQUES ET BIOLOGIQUES DE LA POPULATION D'ALOSE FEINTE DU RHÔNE (ALOSA FALLAX RHODANENSIS ROULE, 1924).
}

\author{
M. LE CORRE (1, 2), J.L. BAGLINIÈRE (1), R. SABATIÉ $(1,2)$, J.Y. MENELLA (3) \\ et D. PONT (4)
}

(1) INRA, Laboratoire d'Ecologie Aquatique, 65 rue de St-Brieuc, 35042 Rennes Cedex, France.

(2) ENSAR, Laboratoire d'Halieutique, 65 rue de St-Brieuc, 35042 Rennes Cedex, France.

(3) Association Migrateurs Rhône-Méditerranée, Z.I. du port fluvial, chemin Segonaux, 13200 Arles, France.

(4) CNRS, Laboratoire d'Ecologie des Systèmes Fluviaux, 1 rue Parmentier, 13200 Arles, France.

\section{RÉSUMÉ}

Les caractéristiques morphologiques et biologiques de la population actuelle d'Alose feinte du Rhône (Alosa fallax rhodanensis) sont présentées. Elles sont analysées à partir d'un lot de 1479 adultes capturés (pêche au filet) lors de leur migration anadrome au cours de trois années.

Les critères morphologiques utilisés (notamment le nombre de branchiospines) et leur faible variabilité individuelle ne font apparaître qu'un seul taxon, identifié comme étant celui d'Alosa fallax rhodanensis.

La longueur moyenne (Lf) est de $433 \mathrm{~mm}$ pour un poids moyen de $1115 \mathrm{~g}$; avec un spectre d'âge s'étalant de 3 à 8 ans. Le rapport des sexes est en moyenne en faveur des femelles (mâles/femelles $=0,61$ ). Celles-ci ont un âge moyen de remontée (AMR) plus élevé $(5,24$ ans) que celui des mâles ( 4,21 ans). A âge égal, les femelles montrent une plus forte croissance linéaire et pondérale que les mâles.

L'âge moyen de première reproduction (AMPR) est plus faible que l'AMR è montre un phénomène d'itéroparité important; il est plus élevé chez les femelles (4,00 ans) que chez les mâles (3,35 ans). Le nombre de reproductions antérieures varie de 1 à 5 et le pourcentage de poissons s'étant déjà reproduits au moins une fois est en moyenne de $48,6 \%$, mais est plus élevé chez les femelles. biologiques.

Il existe des différences inter-annuelles significatives pour la plupart des paramètres

Les résultats obtenus sont discutés et comparés à ceux de DOUCHEMENT (1981) en prenant en compte la part occupée par les méthodes d'échantillonnage dans la variabilité. D'une part, ils confirment certaines caractéristiques biologiques du genre Alosa et du taxon étudié et, d'autre part, ils suggèrent un possible changement de la stratégie démographique de l'Alose du Rhône en 20 ans.

Mots-clés : Alose feinte, morphologie, écologie, adulte, fleuve, Rhône. 


\title{
RECENT DEVELOPMENTS ON BIOLOGICAL AND MORPHOLOGICAL CHARACTERISTICS OF TWAITE SHAD (ALOSA FALLAX RHODANENSIS ROULE, 1924) POPULATION IN THE RIVER RHONE.
}

\begin{abstract}
Morphological and biological characteristics of the present twaite Shad (Alosa fallax rhodanensis) population in the River Rhone (France) are presented. Results are obtained from analysis of a 1479 adults sample collected during upstream migration over three years.
\end{abstract}

Used morphological characters (mainly the number of gillrakers) and their low individual variability reveal that only one taxon is present in the sample and is identified as Alosa fallax rhodanensis.

Mean length (Lf) and weight are $433 \mathrm{~mm}$ and $1115 \mathrm{~g}$, respectively. Age is ranged from 3 to 8 years and sex-ratio is on average in favour of females (males/females $=0.61$ ). Females are older than males (5.24 years against 4.21 years for mean migration age (AMR) and get a better growth in size and weight at the same age.

Mean first reproduction age (AMPR) is lower than AMR because of a large iteroparity phenomenon. AMPR is higher in females ( 4.00 years) than in males (3.35 years). Number of previous spawnings is ranged from 1 to 5 and mean proportion of previous spawners (at least one time) is $48.6 \%$, but it is higher in females.

There exist significant interannual differences in the most biological parameters.

Present results are discussed and compared to these obtained by DOUCHEMENT (1981) by taking into account variability related to sampling methods. On one hand, they confirm some biological characteristics of Alosa genus and studied taxon. On the other hand, they suggest a possible change in demographical strategy of Rhone Shad population in 20 years.

Key-words : twaite Shad, morphology, ecology, adult, river, Rhone.

\section{INTRODUCTION}

Un plan " migrateurs" a été mis en place dans la région Rhône-Méditerranée pour restaurer les populations de grands migrateurs (notamment les aloses et l'anguille). Cette opération de restauration doit aboutir à la reconquête d'une grande partie du fleuve et de ses affluents.

La faisabilité de ce projet s'est trouvée renforcée lorsqu'il a été démontré la possibilité de réhabiliter la libre circulation de l'alose sur le Rhône (LEGAULT, 1993) qui, de fait, est devenue la première espèce cible de ce programme de restauration. Actuellement, les aloses ne colonisent plus que la partie aval du fleuve (aval du barrage de Vallabrègues situé à $50 \mathrm{~km}$ de la mer) alors qu'à la fin du XIXème siècle (BLANCHARD, 1866 ; BRIOCCHI, 1896) et au début du XXème (GALLOIS, 1946) elles étaient très abondantes sur le Rhône, jusqu'au lac du Bourget et sur la plupart de ses grands affluents (Isère, Drôme, Saône). C'est vers le milieu du XXème siècle qu'une régression des populations s'est amorcée sur l'ensemble du fleuve avec la construction des premiers barrages (GALLOIS, 1947), pour s'accentuer fortement avec la poursuite des aménagements du bassin du Rhône (LARINIER et al., 1978).

Dans le cadre de ce plan "migrateurs " multi-partenaires sont prévus à la fois des actions techniques, des travaux d'aménagement et un programme d'études scientifiques. Ce dernier a été mis en place afin d'obtenir des données biologiques sur les populations actuelles d'aloses du Rhône, ce qui constitue un complément indispensable aux travaux d'ingénierie déjà entrepris. Cette acquisition reste nécessaire pour deux raisons : 
- Les aloses de la Méditerranée française, espèces anadromes appartenant au groupe géographique de l'Atlantique-est et de la Méditerranée, ont une position taxonomique très confuse. Leurs critères méristiques les rattachent au taxon Alosa fallax (QUIGNARD et KARTAS, 1977 ; WHITEHEAD, 1985). Cependant, leurs caractéristiques écologiques (taille moyenne plus élevée que l'Alose feinte, longue distance de migration vers les parties hautes des cours d'eau) les rapprochent de l'autre taxon Alosa alosa (grande Alose) (HOESTLANDT, 1958). Ces deux observations montrent le caractère particulier de ces aloses et expliquent les changements ou les incertitudes dans leur position taxonomique. En effet, ROULE (1925) a considéré l'Alose du Rhône comme une espèce à part entière tandis que QUIGNARD et KARTAS (1977), à partir d'une étude approfondie des critères méristiques, ont placé ces poissons au rang de sousespèce (Alosa fallax rhodanensis) en la dissociant des taxons $A$. f. fallax et $A$. f. nilotica, présents respectivement en Atlantique et en Méditerranée orientale. Cette nomenclature semble être celle qui est reprise actuellement le plus fréquemment (QUIGNARD et DOUCHEMENT, 1991), même si WHITEHEAD (1985), dans sa classification taxonomique du genre Alosa, et BIANCO (1995) ne considèrent l'existence que d'un taxon méditerranéen.

Deux autres éléments s'ajoutent à la difficulté du positionnement taxonornique de ces espèces :

1 - L'incertitude concernant la présence de la grande Alose sur le Rhône. ROULE (1924), GALLOIS (1946) et HOESTLANDT (1958) l'ont toujours considérée comme très douteuse. Cependant, HOESTLANDT (1958), en réalisant un transfert qu'il considère comme réussi entre la Dordogne et l'Ardèche, a pu modifier la situation initiale. Mais aucun suivi à long terme de cette opération n'a été effectué. Cependant, certaines observations (ANONYME; 1975 ; DOUCHEMENT, 1981) mentionnent la présence de grandes aloses dans le Rhône au début de la décennie 1970, puisque des individus capturés à cette époque présentaient un nombre de branchiospines comparable à celui généralement comptabilisé dans les populations de l'Atlantique-est (> 90) (ANONYME, 1975; TAVERNY, 1991 ; QUIGNARD et DOUCHEMENT, 1991 ; SABATIE, 1993 ; ALEXANDRINO, 1996). En revanche, DOUCHEMENT (1981) a considéré avoir détecté la présence de la grande Alose dans le Rhône et l'Aude à partir d'individus dont le nombre moyen de branchiospines (entre 70 et 72 ) était bien inférieur à celui évoqué précédemment.

2 - La présence d'individus hybrides entre la grande Alose et l'Alose feinte dans la population du Rhône (DOUCHEMENT, 1981). Ce phénomène d'hybridation, observé sur certains bassins versants de leur aire de répartition (MENNESSON-BOISNEAU et BOISNEAU, 1990 ; SABATIE, 1993; ALEXANDRINO, 1996), a été confirmé récemment par une étude génétique (ALEXANDRINO et al., 1996).

- Les connaissances écologiques étant anciennes (ROULE, 1925 ; GALLOIS, 1946) et limitées (DOUCHEMENT, 1981), il convient de les actualiser et de les élargir afin de pouvoir optimiser ce programme de restauration.

Le présent article apporte les premiers éléments de caractérisation biologique et taxonomique des aloses du Rhône acquis dans le cadre de ce programme à partir d'échantillons d'aloses récoltés en 1994, 1995 et 1996. Les résultats sont comparés à ceux obtenus par DOUCHEMENT (1981) dans le même secteur géographique à peu près 20 ans auparavant.

\section{MATÉRIEL ET MÉTHODES}

\section{Récolte des données}

La population d'aloses adultes a été échantillonnée au cours de leur migration de reproduction (de mi-mars à fin juin) durant trois années :

- 1994 : du 5 mai au 23 juin ;

- $1995:$ du 7 avril au 19 juillet

- 1996 : du 29 mai au 20 juin (récolte de quelques spécimens auprès des pêcheurs du 23 avril au 29 mai). 


\section{Figure 1}

Site d'étude (* : site d'échantillonnage).

\section{Figure 1}

Studied area (* : sampling location).

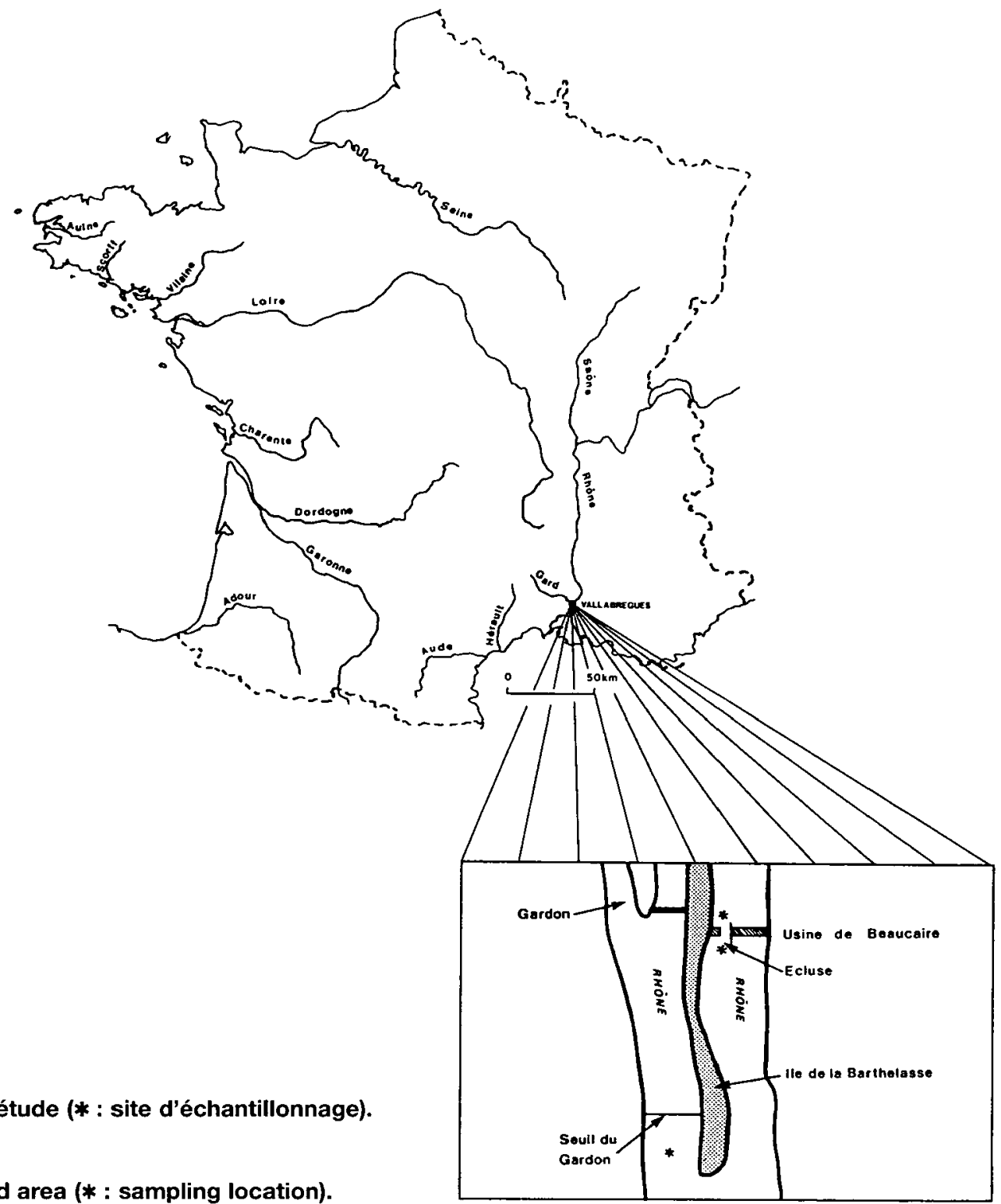

Les aloses adultes ont été capturées à l'aide de filets (maillant fixe de maille* $80,90,100$ et $120 \mathrm{~mm}$, et maillant dérivant ou droit de maille 70,90 et $120 \mathrm{~mm}$ ) en amont et en aval du barrage de Vallabrègues, lors des périodes d'éclusée permettant le passage des poissons (Fig. 1).

739, 424 et 316 aloses ont été capturées respectivement en 1994, 1995 et 1996 (Tableau I). La taille (Lf en mm pour les trois années et Lt uniquement en 1995 et 1996), le poids (en g) et le sexe ont été notés sur la quasi-totalité des poissons capturés. Des écailles ont été prélevées sur $181(24,5 \%), 253(57,1 \%)$ et 314 (99 \%) individus respectivement en 1994, en 1995 et 1996. Les données de types morphométrique et méristique, considérées comme les plus pertinentes pour identifier les taxons du genre Alosa (MENNESSON-BOISNEAU et BOISNEAU, 1990; SABATIE, 1993 ; ALEXANDRINO, 1996 ; Tableau I), ont été recueillies sur un petit nombre de poissons en 1995 et sur tous les individus en 1996.

* La taille des mailles correspond au maillage étiré. 
Tableau I

Données recueillies sur le terrain ( $\mathrm{N}:$ nombre d'individus).

Table I

Data collected in the field ( $\mathbf{N}$ : number of individuals).

\begin{tabular}{|c|c|c|c|c|c|}
\hline type de données & variable & définition & N 1994 & N 1995 & N 1996 \\
\hline \multirow{3}{*}{ biométrique } & Lf & longueur à la fourche $(\mathrm{mm})$ & 737 & 424 & 314 \\
\hline & $\mathrm{Lt}(1995)$ & longueur totale $(\mathrm{mm})$ & 0 & 424 & 316 \\
\hline & poids & poids frais $(\mathrm{g})$ & 615 & 424 & 311 \\
\hline \multirow{6}{*}{ méristique } & $\mathrm{Br}$ & $\begin{array}{l}\text { nombre de branchiospines sur le } \\
\text { premier arc branchial gauche }\end{array}$ & 0 & 32 & 316 \\
\hline & RDal & $\begin{array}{l}\text { nombre de rayons sur la } \\
\text { nageoire dorsale }\end{array}$ & 0 & 43 & 314 \\
\hline & RAal & $\begin{array}{l}\text { nombre de rayons sur la } \\
\text { nageoire anale }\end{array}$ & 0 & 43 & 314 \\
\hline & Scut ant & nombre de scutelles antérieures & 0 & 10 & 315 \\
\hline & Scut post & nombre de scutelles postérieures & 0 & 11 & 315 \\
\hline & Scut tot & nombre total de scutelles & 0 & 10 & 315 \\
\hline
\end{tabular}

\section{Traitement des données}

\section{Estimation de l'âge}

Les écailles récoltées ont été triées, les 4 à 5 meilleures ont été mises à tremper dans une solution de soude $(5 \%)$ pendant 5 minutes puis rincées à l'eau distillée, nettoyées et séchées avant d'être montées entre lame et lamelle. La lecture a été effectuée à l'aide d'un lecteur de microfiches. L'interprétation des écailles a été faite en suivant les recommandations de MENNESSON-BOISNEAU et BAGLINIĖRE (1992). Quand les lignes d'arrêt de croissance étaient difficiles à localiser sur les écailles, elles ont été positionnées en tenant compte du nombre de stries transversales (DOUCHEMENT, 1981). Le nombre d'écailles non interprétables et/ou régénérées était très faible $(1,9 \%)$.

\section{Structure de la population adulte}

Une régression linéaire entre les longueurs totale $(\mathrm{Lt}$ en $\mathrm{mm})$ et à la fourche ( $\mathrm{Lf}$ en $\mathrm{mm})$ a été calculée afin de pouvoir comparer les tailles de la population avec celles données par DOUCHEMENT (1981) (uniquement longueur totale); cette relation est très étroite et s'exprime par la formule suivante: $L t=1,0315 \mathrm{Lf}+41,76\left(n=739 ; r^{2}=0,95(p<0,01)\right)$. De même, une relation entre la taille ( $L f$ ou $L t$ en $\mathrm{mm}$ ) et le poids ( $\mathrm{P}$ en $\mathrm{g}$ ) de type puissance a été établie pour les trois années par transformation logarithmique des données.

Les distributions de tailles de la population totale capturée et des individus sur lesquels les écailles ont été prélevées ont été comparées afin d'analyser la représentativité de l'échantillon “ écaille " pour les deux premières années de l'étude (1994 et 1995).

La mesure de la taille et l'observation du sexe des poissons d'une part et l'estimation de l'âge d'autre part ont permis d'aborder la structure démographique de la population de géniteurs capturée avec : 
- la taille moyenne par âge et par sexe;

- le rapport des sexes (nombre de mâles/nombre de femelles) uniquement en 1994 et 1995, car en 1996 l'échantillonnage a été mené de façon à obtenir autant de mâles que de femelles ;

- l'âge moyen de remontée (AMR) et celui de première reproduction (AMPR) pour la population de géniteurs et celle des deux sexes. Ces deux paramètres ont été calculés selon la formule de FAHY (1978) :

$$
A m=S \underset{i=n}{i=1} \times \text { ni.ai } / S \text { ni }
$$

avec ai $=$ classe d'âge $\mathbf{i}$ et $n i=$ nombre d'individus d'âge $\mathbf{i}$;

- le nombre de reproductions.

Méristique et morphométrie

La valeur moyenne des variables méristiques et le coefficient de variation (cv) ont été calculés. Une régression linéaire a été établie entre le nombre de branchiospines $(\mathrm{Br})$ et la longueur totale $(\mathrm{Lt})$ des individus.

Le traitement et la comparaison des données ont été effectués à l'aide de tests statistiques classiques (t de Student, $\chi^{2}$, régression linéaire et analyse de variance).

\section{RÉSULTATS}

\section{Caractéristiques morphologiques}

La taille $(L t)$ des individus dont les caractéristiques morphologiques ont été relevées varie entre 300 et $625 \mathrm{~mm}$.

\section{Tableau II}

Moyenne des valeurs des différentes variables méristiques prises en compte ( $N$ : nombre d'individus, min-max : minimum et maximum, cv : coefficient de variation, entre parenthèses : valeur donnée par DOUCHEMENT, 1981).

\section{Table II}

Average of the different meristic variables ( $N$ : number of individuals, $\min -\max : \operatorname{minimum}$ and maximum, cv : coefficient of variation, in brackets : data by DOUCHEMENT, 1981).

\begin{tabular}{cccccc}
\hline variable & $\mathrm{N}$ & moyenne & écart-type & min-max & cv \\
\hline Br & $345(380)$ & $38,38(39,85)$ & $2,09(2,32)$ & $31-46$ & 0,005 \\
RDal & $356(646)$ & $18,92(19,61)$ & $0,79(0,78)$ & $15-22$ & 0,042 \\
RAal & $356(642)$ & $21,91(22,54)$ & $1,04(1,07)$ & $18-25$ & 0,047 \\
Scut ant & $323(644)$ & $20,97(21,07)$ & $0,87(0,91)$ & $15-24$ & 0,041 \\
Scut post & $324(644)$ & $15,12(15,54)$ & $0,89(0,8)$ & $10-17$ & 0,059 \\
Scut tot & $323(644)$ & $36,10(36,61)$ & $1,29(1,06)$ & $30-40$ & 0,036 \\
\hline
\end{tabular}


La faible amplitude des valeurs extrêmes et les valeurs réduites du coefficient de variation des caractéristiques morphologiques traduisent une forte homogénéité de la population échantillonnée (valeurs moyennes données dans le tableau II).

Le nombre de branchiospines $(\mathrm{Br})$ est corrélé significativement avec la taille du poisson (Lf) en s'exprimant par la relation: $B r=0,007 \mathrm{Lf}+35,586\left(n=346, r^{2}=0,03\right.$ et $\left.p<0,01\right)$; il n'augmente que très légèrement avec celle-ci (Fig. 2), ce qui justifie de présenter la valeur moyenne du caractère.

$$
\begin{gathered}
\mathrm{Br}=0,007 \mathrm{Lf}+35,586 \\
\mathbf{r}^{2}=0,03 \text { et } \mathrm{N}=346
\end{gathered}
$$

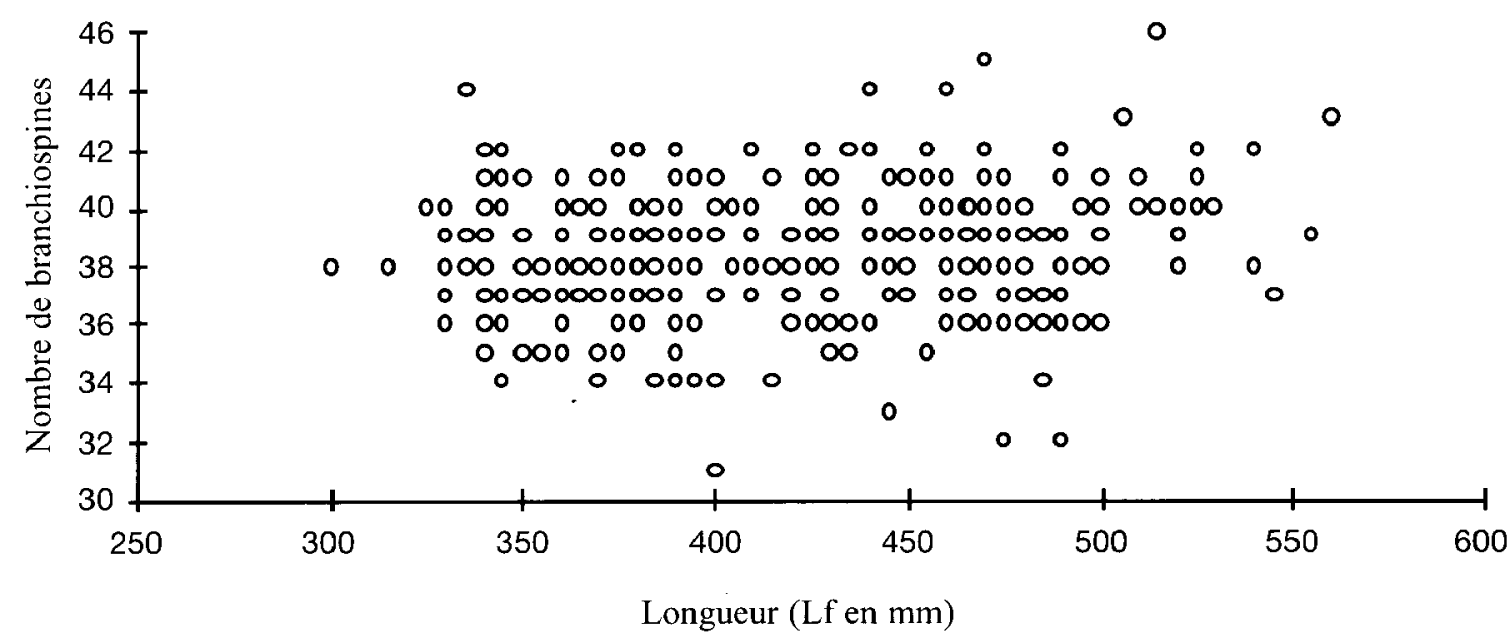

Figure 2

Relation entre le nombre de branchiospines (Br) et la taille ( $\mathrm{Lf}$ en $\mathrm{mm}$ ) des aloses échantillonnées sur le Rhône en 1995 et 1996 ( $N$ : nombre d'individus).

Figure 2

Relation between the number of gillrakers $(\mathrm{Br})$ and the fork length ( $\mathrm{Lf}$ in $\mathrm{mm}$ ) of the shads sampled in the River Rhone in 1995 and 1996 ( $N$ : individuals number).

\section{Caractéristiques biologiques}

Taille, poids et rapport des sexes

L'analyse des caractéristiques biométriques générales et du rapport des sexes montre que :

- Le spectre de taille et de poids de la population globale échantillonnée varie de 135 à $595 \mathrm{~mm}$ (Lf) et de 110 à 3010 grammes (Tableau III), et reste très semblable lors des trois campagnes. Cependant, les distributions de taille et de poids diffèrent notablement entre les trois années, en raison d'une proportion plus élevée de poissons de grande taille capturés en 1995 (Fig. 3). 


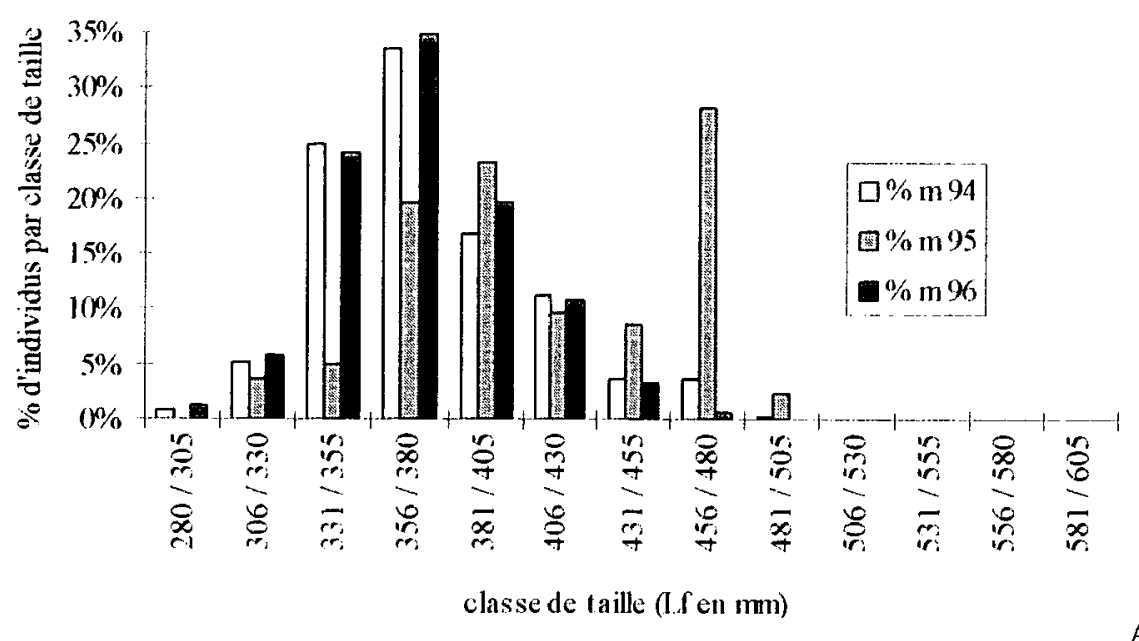

A

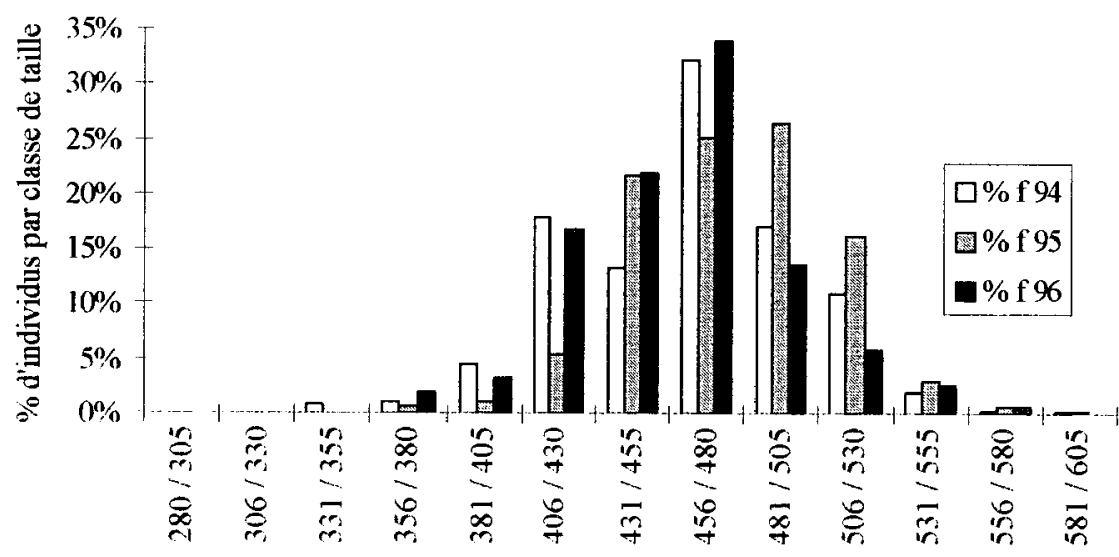

classe de taille (If en mm)

B

Figure 3

Répartition des tailles (Lf en mm) des aloses capturées sur le Rhône en 1994, 1995 et 1996 (A : mâles, $m$ : mâle ; $B$ : femelles, $f$ : femelle).

Figure 3

Lengths repartition (Lf in $\mathrm{mm}$ ) of shads caught in the River Rhone in 1994, 1995 and 1996 (A : males, $m$ : male ; $B$ : females, $f$ : female).

- Le rapport des sexes est globalement en faveur des femelles $(0,61)$ mais diffère significativement selon l'année $(p<0,001)$; il est équilibré en 1994 et 1996 , et très fortement en laveur des femelles en 1995 (Tableau III). La taille et le poids moyens des femelles sont supérieurs à ceux des mâles $(p<0,001)$, quelle que soit l'année. Ces trois observations expliquent la difference de distribution des tailles el des poids constatée entre les trois échantillons.

- La taille et le poids sont très fortement corrélés aussi bien pour la population totale que pour chacun des sexes, quelle que soit l'année (Tableau III). Cependant, la valeur du coefficient de détermination ( $r$ ?) apparaît moins élevée chez les mâles en 1994 et 1996, et chez les femelles en général. L'expression de ces relations montre qu'à taille égale, les femelles sont plus lourdes que les mâles. 


\section{Tableau III}

Caractéristiques biométriques générales et rapport des sexes des aloses adultes capturées sur le Rhône en 1994, 1995 et 1996 ( $N$ : nombre d'individus, $\sigma$ : écart-type, $r^{2}$ : coefficient de détermination).

\section{Table III}

Biometric characteristics and sex-ratio of adult shads caught in the River Rhone in 1994, 1995 and 1996 ( $N$ : number of individuals, $\sigma$ : standard deviation, $r^{2}$ : coefficient of determination).

\begin{tabular}{|c|c|c|c|c|c|}
\hline & & & 1994 & 1995 & 1996 \\
\hline \multirow{6}{*}{$\begin{array}{c}\text { longueur } \\
\text { (Lf en mm) }\end{array}$} & \multirow{3}{*}{ mâles } & moyenne & 376 & 413 & 373 \\
\hline & & $(\mathrm{N} ; \sigma)$ & $(357 ; 37,2)$ & $(82 ; 47,4)$ & $(158 ; 31,07)$ \\
\hline & & extrêmes & $135-485$ & $315-500$ & $255-470$ \\
\hline & \multirow{3}{*}{ femelles } & moyenne & 464 & 478 & 460 \\
\hline & & $(\mathrm{N} ; \sigma)$ & $(382 ; 39,1)$ & $(342 ; 33,2)$ & $(157 ; 35,3)$ \\
\hline & & extrêmes & $335-595$ & $365-590$ & $360-560$ \\
\hline \multirow{6}{*}{$\begin{array}{l}\text { poids } \\
(\mathrm{P} \text { en } \mathrm{g})\end{array}$} & \multirow{3}{*}{ mâles } & moyenne & 672 & 914 & 606 \\
\hline & & $(\mathrm{N} ; \sigma)$ & $(316 ; 209,5)$ & $(82 ; 335)$ & $(156 ; 158,2)$ \\
\hline & & extrêmes & $335-1540$ & $362-1627$ & $367-1106$ \\
\hline & \multirow{3}{*}{ femelles } & moyenne & 1347 & 1524 & 1286 \\
\hline & & $(\mathrm{N} ; \sigma)$ & $(300 ; 382)$ & $(342 ; 317,7)$ & $(1516 ; 353,2)$ \\
\hline & & extrêmes & $110-2500$ & $542-2380$ & $566-3010$ \\
\hline \multirow{4}{*}{\multicolumn{2}{|c|}{$\begin{array}{l}\text { relation taille (Lf)-poids } \\
\text { (P) }\left(\mathrm{N} ; \mathrm{r}^{2}\right)\end{array}$}} & mâles & $P=9.10^{-5} \mathrm{Lf} 2,66$ & $\mathrm{p},=1.10^{-6} \mathrm{Lf}^{3,42}$ & $P=6.10^{-4} L f 2,34$ \\
\hline & & & $\left(314 ; 0,721 \mathrm{p}<10^{-2}\right)$ & $\left(79 ; 0,916 p<10^{-2}\right)$ & $\left(156 ; 0,670 \mathrm{p}<10^{-2}\right)$ \\
\hline & & femelles & $\begin{array}{c}P=2.10^{-4} L^{2} 2,54 \\
\left(294 ; 0,544 p<10^{-2}\right)\end{array}$ & $\begin{array}{c}\mathrm{P}=2.10^{-4} \mathrm{Lf} 2,58 \\
\left(342 ; 0,674 \mathrm{p}<10^{-2}\right)\end{array}$ & $\begin{array}{c}\mathrm{P}=1.10^{4} \mathrm{Lf}_{\mathrm{f}} 2,66 \\
\left(154 ; 0,590 \mathrm{p}<10^{-2}\right)\end{array}$ \\
\hline & & $\begin{array}{l}\text { population } \\
\text { totale }\end{array}$ & $\begin{array}{c}P=9.10^{-6} \mathrm{Lf}^{3}, 05 \\
\left(611 ; 0,835 \mathbf{p}<10^{-2}\right)\end{array}$ & $\begin{array}{c}\mathrm{P}=3.10^{-6} \mathrm{Lf}^{3,23} \\
\left(421 ; 0.845 \mathrm{p}<10^{-2}\right)\end{array}$ & $\begin{array}{c}P=4.10^{-6} \mathrm{Lf} 3,17 \\
\left(310 ; 0,860 \mathrm{p}<10^{-2}\right)\end{array}$ \\
\hline \multicolumn{3}{|c|}{ rapport des sexes (mâles / femelles) } & 0,93 & 0,24 & 1,00 \\
\hline
\end{tabular}

\section{Structure d'âge}

La répartition en taille de la population totale et celle des individus sur lesquels des écailles ont été prélevées ne montrent aucune différence significative $\left.\chi^{2}=N S, p>0,05\right)$. Ainsi, les résultats obtenus à partir de l'échantillon " écaille " restent représentatifs de l'ensemble de la population d'aloses échantillonnée au cours des campagnes. L'analyse de cet échantillon fait ressortir que :

- Le spectre de classe d'âge de la population de géniteurs varie de 3 à 8 ans pour les femelles et les mâles, mais il peut varier en fonction des années (Fig. 4).

- La répartition des classes d'âge est différente selon l'année et le sexe. La population échantillonnée est en moyenne plus âgée en 1995 et les femelles sont toujours plus âgées que les mâles. Ainsi, 63,6\% des mâles appartiennent aux classes d'âge de 3 et 4 ans en 1994 alors que $74,5 \%$ et $79,1 \%$ appartiennent à celles de 4 et 5 ans respectivement en 1995 et 1996 . De plus, $74,4 \%$ et $70,9 \%$ des femelles sont âgées de 4 à 5 ans respectivement en 1994 et 1996 alors que $82 \%$ d'entre elles ont 5 et 6 ans en 1995 (Fig. 4). Cette différence de répartition selon le sexe et l'année s'exprime également au travers des valeurs de l'âge moyen de remontée des géniteurs (AMR) ; ce dernier est identique en $1994(4,52)$ et $1996(4,57)$, mais plus faible qu'en $1995(5,34)$; il est toujours plus élevé chez les femelles que chez les mâles (Tableau IV). Sur les trois années, l'AMR est en moyennè de 4,82 ans pour la population de géniteurs, de 4,21 ans pour les mâles et de 5,24 ans pour les femelles. 


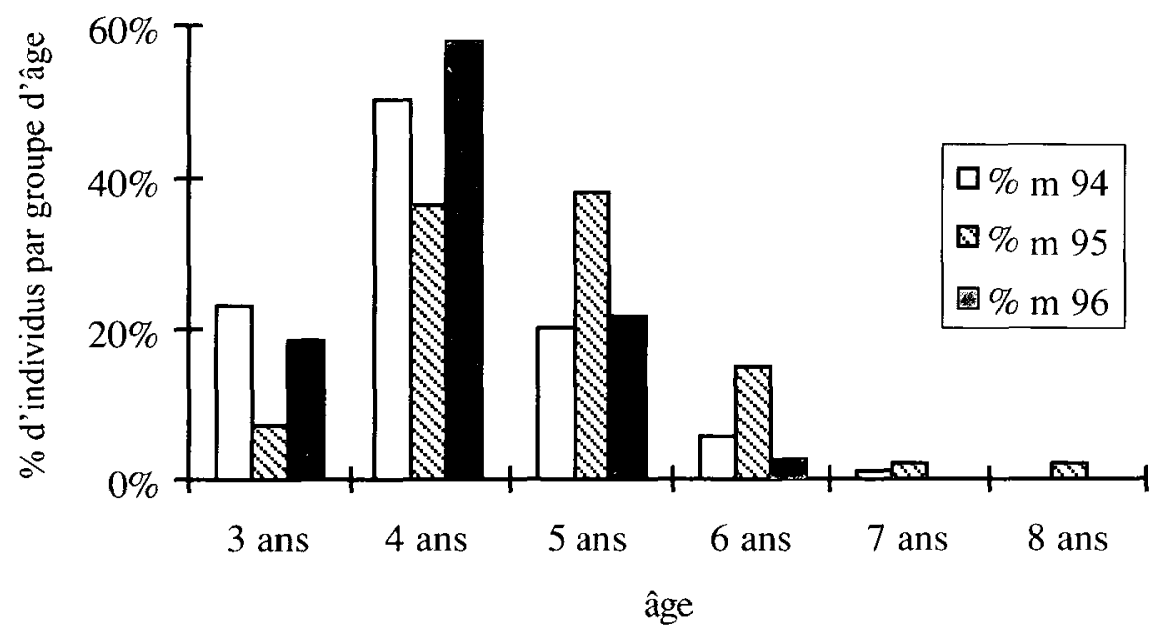

A

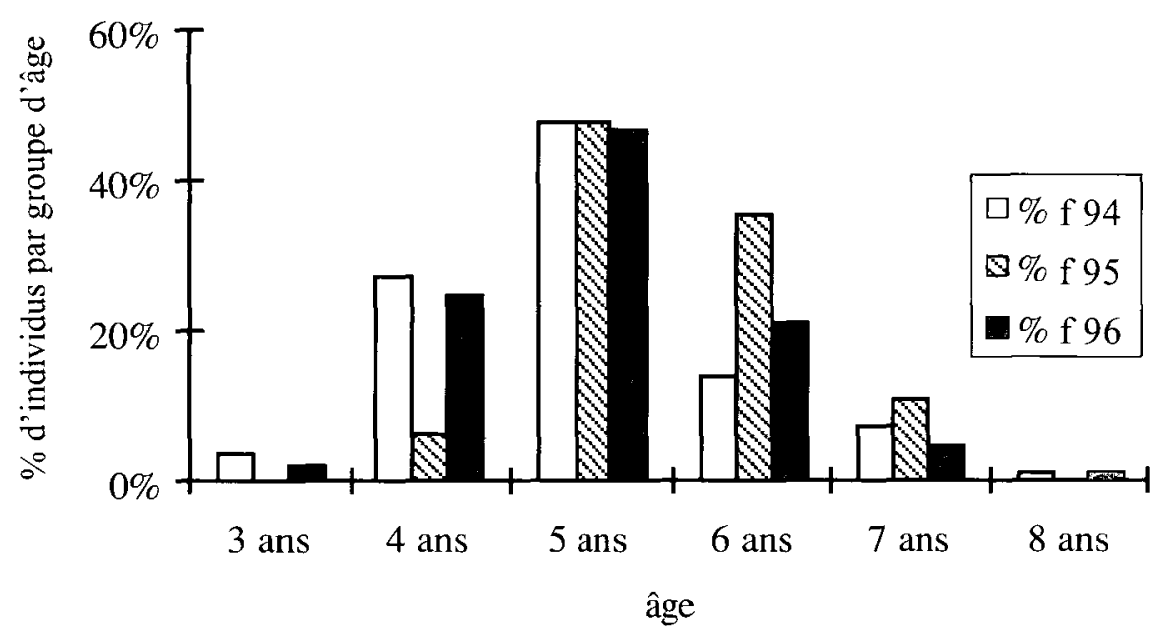

B

Figure 4

Répartition (\%) par classe d'âge des aloses mâles (A, $m$ : mâle) et femelles (B, $f:$ femelle) échantillonnées sur le Rhône en 1994, 1995 et 1996.

Figure 4

Repartition (\%) per age range of the male (A, $m$ : male) and female (B, $f:$ female) shads caught in the River Rhone in 1994, 1995 and 1996.

- A âge égal, les femelles ont toujours une taille et un poids moyens supérieurs à ceux des mâles (Tableau IV). II existe des différences significatives entre les tailles moyennes annuelles des classes d'âge pour les deux sexes. Ainsi, chez les mâles, les individus de 5 ans $\left(r^{2}=0,34\right)$ et 6 ans $\left(r^{2}=0,83\right)$ sont significativement $(p<0,001)$ plus petits en 1996 qu'en 1994 et 1995 (Tableau IV). En revanche, les individus de 3 ans sont significativement $(p=0,023)$ plus grands en 1996 ; cependant, en ce qui concerne cette dernière classe d'âge, l'effectif du souséchantillon 1995 est très faible comparativement à ceux des deux autres années (Tableau IV). Chez les femelles, les différences inter-annuelles sont moins marquées. En effet, si des différences significatives apparaissent pour les individus âgés de 4 ans $\left(r^{2}=0,1\right.$ et $\left.p=0,02\right)$, 5 ans $\left(r^{2}=0,03\right.$ et $\left.p=0,03\right)$ et 6 ans $\left(r^{2}=0,09\right.$ et $\left.p=0,006\right)$, le pourcentage de variance expliqué par "l'effet année " est faible. 


\section{Tableau IV}

Taille (Lf en $\mathrm{mm}$ ) et poids (P en g) moyens par classe d'âge. Ages moyens de remontée (AMR) et de première reproduction (AMPR) des aloses adultes capturées sur le Rhône en 1994, 1995 et 1996 ( $N$ : nombre d'individus ; $\sigma$ : écart-type).

\section{Table IV}

Average length (Lf in $\mathrm{mm}$ ) and average weight $(P$ in $g$ ) for each age class. Average age of upstream migration (AMR) and average age of the first spawning (AMPR) of adult shads caught in the River Rhone in 1994, 1995 and 1996 (N : number of individuals, $\sigma$ : standard deviation).

\begin{tabular}{|c|c|c|c|c|c|c|c|}
\hline & & \multicolumn{2}{|c|}{1994} & \multicolumn{2}{|c|}{1995} & \multicolumn{2}{|c|}{1996} \\
\hline & & mâles & femelles & mâles & femelles & mâles & femelles \\
\hline \multirow{2}{*}{3} & $\operatorname{Lf}(N ; \sigma)$ & $342(22 ; 21,2)$ & $372(3 ; 27,5)$ & $335(4 ; 9,1)$ & (0) & $361(28 ; 31,3)$ & $413(3 ; 24,7)$ \\
\hline & $P(N ; \sigma)$ & $538(22 ; 61,7)$ & $747(3 ; 246,1)$ & $437(4 ; 53,7)$ & (0) & $556(27 ; 143)$ & $1086(3 ; 172,2)$ \\
\hline \multirow[t]{2}{*}{4} & $\operatorname{Lf}(N ; \sigma)$ & $379(48 ; 23,3)$ & $424(23 ; 21,4)$ & $375(20 ; 23,9)$ & $434(12 ; 24,4)$ & $368(88 ; 27,6)$ & $444(38 ; 30)$ \\
\hline & $P(N ; \sigma)$ & $708(44: 129,9)$ & $1094(21 ; 228,7)$ & $630(20 ; 130,4)$ & $1156(12 ; 234,5)$ & $573(88 ; 129,3)$ & $1137(38 ; 314)$ \\
\hline \multirow[t]{2}{*}{5} & $\operatorname{Lf}(N ; \sigma)$ & $435(18 ; 24,7)$ & $469(41 ; 29,7)$ & $434(21 ; 29,8)$ & $465(94 ; 24,4)$ & $392(33 ; 33,0)$ & $456(71 ; 29,8)$ \\
\hline & $\mathrm{P}(\mathrm{N} ; \sigma)$ & $1034(18 ; 220,6)$ & $1476(39 ; 378,3)$ & $1067(21 ; 300,7)$ & $1411(94 ; 216,7)$ & $739(32 ; 184,0)$ & $1231(7 \mathrm{I} ; 272,3)$ \\
\hline \multirow[t]{2}{*}{6} & $\operatorname{Lf}(N ; \sigma)$ & $454(5 ; 15,17)$ & $506(12 ; 30,5)$ & $459(8 ; 14,5)$ & $495(70 ; 25,4)$ & $394(4 ; 7,5)$ & $480(32 ; 29,8)$ \\
\hline & $\mathrm{P}(\mathrm{N} ; \sigma)$ & $1147(5 ; 138,0)$ & $1686(11 ; 227,4)$ & $1147(8 ; 244,1)$ & $1699(70 ; 255,0)$ & $662(4 ; 62,1)$ & $1498(3 \mathrm{I} ; 389,2)$ \\
\hline \multirow[t]{2}{*}{7} & $\operatorname{Lf}(\mathbb{N} ; \sigma)$ & $480(1 ; 0)$ & $510(6 ; 51,0)$ & $480(1)$ & $506(21 ; 34,7)$ & (0) & $516(7 ; 17,7)$ \\
\hline & $\mathrm{P}(\mathrm{N} ; \sigma)$ & $1540(1 ; 0)$ & $1357(4 ; 786,3)$ & $1329(1)$ & $1762(21 ; 325,0)$ & (0) & $1634(7 ; 230,1)$ \\
\hline \multirow[t]{2}{*}{8} & $\operatorname{Lf}(N ; \sigma)$ & (0) & $520(1 ; 0)$ & $470(1 ; 0)$ & (0) & (0) & $530(2 ; 42,4)$ \\
\hline & $P(N ; \sigma)$ & (0) & $2300(1 ; 0)$ & $1007(1 ; 0)$ & (0) & (0) & $2103(2 ; 497,8)$ \\
\hline & AMR & 4,1 & 5 & 4,7 & 5,5 & 4,1 & 5,1 \\
\hline & AMPR & 3,5 & 4 & 4 & 4,4 & 3,3 & 4 \\
\hline
\end{tabular}

- Les marques de reproduction sont facilement et parfaitement identifiables sur les écailles. Elles se présentent sous la forme d'une ligne continue très distincte faisant le tour de la partie de l'écaille enfoncée dans le derme, voire même de sa partie extérieure (Fig. 5). Autour de cette ligne, il existe une très forte désorganisation des circuli. Cette dernière est due à l'importance du phénomène d'érosion du bord de l'écaille constatée lors de la remontée et liée à la maturation sexuelle. Ce phénomène peut entraîner dans certains cas le télescopage de deux marques de reproduction successives sur les bords latéraux de l'écaille.

- Le taux de poissons s'étant déjà reproduits au moins une fois est en moyenne de $48,6 \%$ dans la population capturée au cours des trois campagnes. Cette proportion est équivalente quelle que soit l'année $\left(\chi^{2}\right.$ non significatif), aussi bien pour la population totale ( $43,8 \%$ en $1994,54,5 \%$ en 1995 et $46,7 \%$ en 1996) que pour chacun des sexes (mâles : $35,8 \%$ en $1994,43,6 \%$ en 1995 et $33,3 \%$ en 1996 ; femelles : $52,3 \%$ en $1994,57,5 \%$ en 1995 et $60 \%$ en 1996). En revanche, la proportion de poissons d'une ou plusieurs remontées est toujours plus élevée chez les femelles $(p<0,01)$.

Ainsi, l'âge moyen de première reproduction (AMPR) est bien inférieur à celui de remontée (AMR) (Tableau IV). Sur les trois années, ce dernier est globalement de 3,81 pour la population de géniteurs, de 3,35 pour celle des mâles et de 4,00 pour celle des femelles. Chez les mâles, 1,5\% de la population se reproduit pour la première fois à l'âge de 2 ans et $90,4 \%$ à 3 et 4 ans. En revanche, chez les femelles, 10,2\% fraient une première fois à l'âge de 3 ans et $85,9 \%$ à 4 et 5 ans (Tableau V ; Fig. 6). La taille de première maturité sexuelle varie de 255 (300 en Lt) à 490 mm (550 en Lt) chez les mâles et de 335 (384 en Lt) à 520 mm (570 en Lt) chez les femelles. 

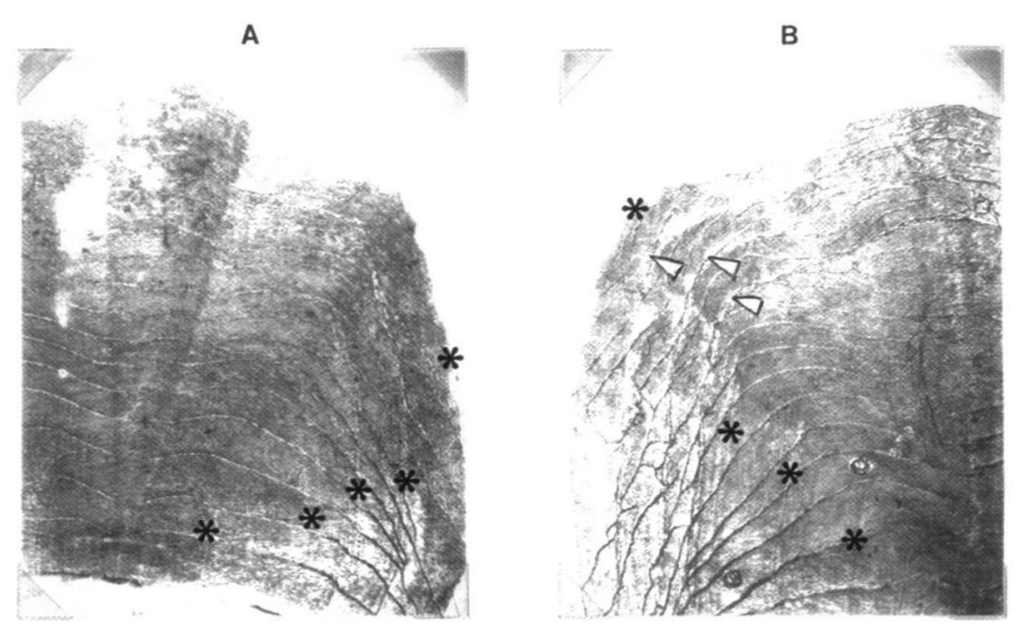

Figure 5

Ecailles d'aloses du Rhône.

A - Individu femelle (Lt = $520 \mathrm{~mm}$; Poids = $1143 \mathrm{~g}$ ) âgé de 5 ans (marque hivernale *) et ne s'étant pas encore reproduit.

B - Individu femelle ( $L t=585 \mathrm{~mm}$; Poids $=2130 \mathrm{~g}$ ) âgé de 7 ans et s'étant déjà reproduit trois fois (marque de reproduction $\Delta$ ).

\section{Figure 5}

Scales of shads from the River Rhone.

A - Female ( $\mathrm{Lt}=520 \mathrm{~mm}$; Weight $=1143 \mathrm{~g})$ of 5 years old (winter mark $*$ ) and which has not yet spawned.

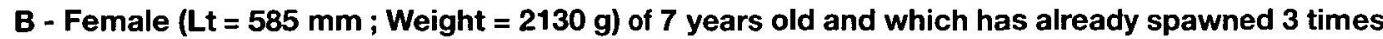
(reproductive mark $\Delta$ ).

\section{Tableau V}

Répartition (\%) par groupe d'âge de première reproduction des aloses capturées sur le Rhône en 1994, 1995 et 1996.

\section{Table V}

Repartition (\%) per average range of the first spawning shads caught in the River Rhone in 1994, 1995 and 1996.

\begin{tabular}{|c|c|c|c|c|c|c|}
\hline \multirow{2}{*}{ sexe } & \multirow{2}{*}{ année } & \multicolumn{5}{|c|}{ âge de première reproduction } \\
\hline & & 2 & 3 & 4 & 5 & 6 \\
\hline répartition (\%) & 1994 & 1,1 & 47,9 & 51 & 0 & 0 \\
\hline des mâles par & 1995 & 0 & 23,2 & 50 & 25 & 1,8 \\
\hline groupe d'âge & 1996 & 2,6 & 37,3 & 53,6 & 6,5 & 0 \\
\hline \multicolumn{2}{|c|}{ moyenne sur les trois années } & 1,5 & 38,3 & 52,1 & 7,6 & 0,3 \\
\hline répartition (\%) & 1994 & 0 & 14,7 & 66,7 & 18,6 & 0 \\
\hline des femdles par & 1995 & 0 & 6,1 & 50,8 & 37,5 & 5,6 \\
\hline groupe dâge & 1996 & 0 & 12,9 & 54,2 & 28,4 & 4,5 \\
\hline \multicolumn{2}{|c|}{ moyenne sur les trois années } & 0 & 10,2 & 54,2 & 31,7 & 3,8 \\
\hline
\end{tabular}



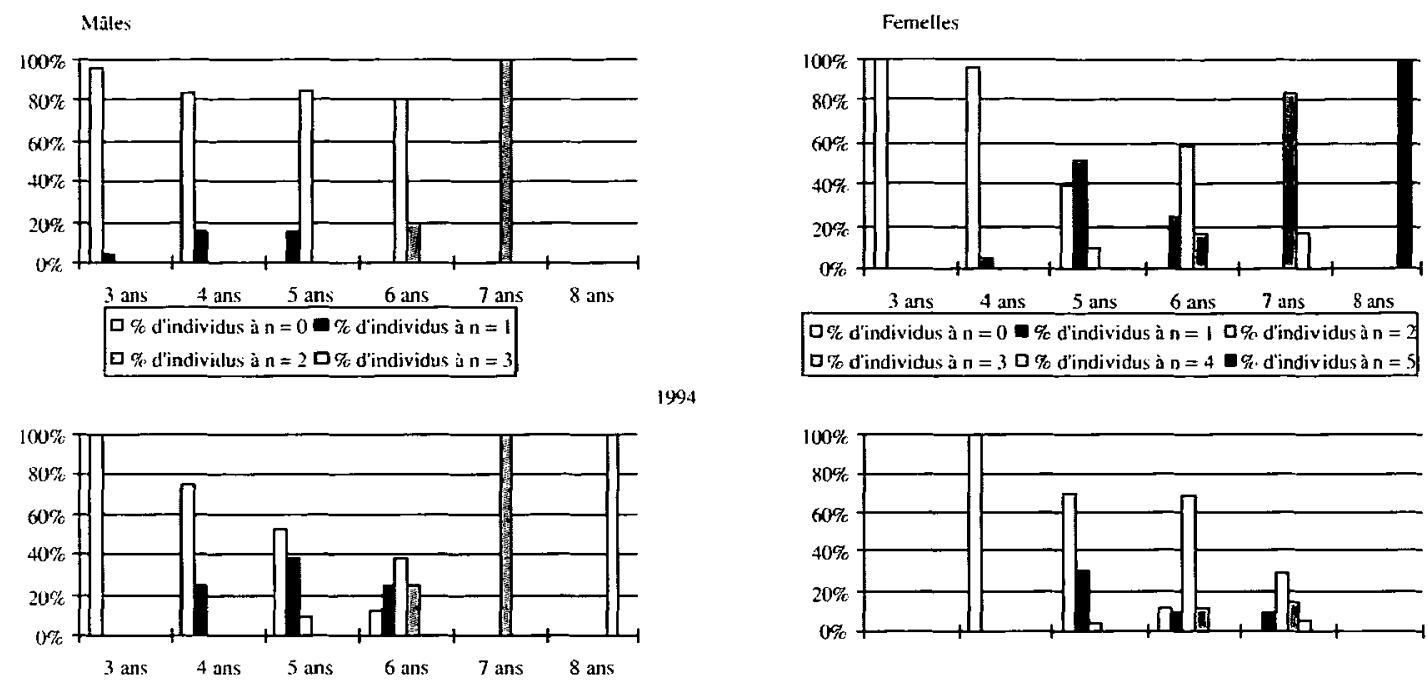

口出 dindividus à $n=0 \quad$ \% d'individus à $n=1 \quad \square \%$ d'individus à $n=$

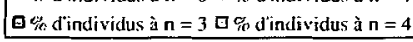

口\% d'individus à $n=0$ Q $\%$ d'individus à $n=1 \quad 0 \%$ d'individus à $n=2$ $\square \%$ d'individus à $n=3 \square \%$ dindividus à $n=4$
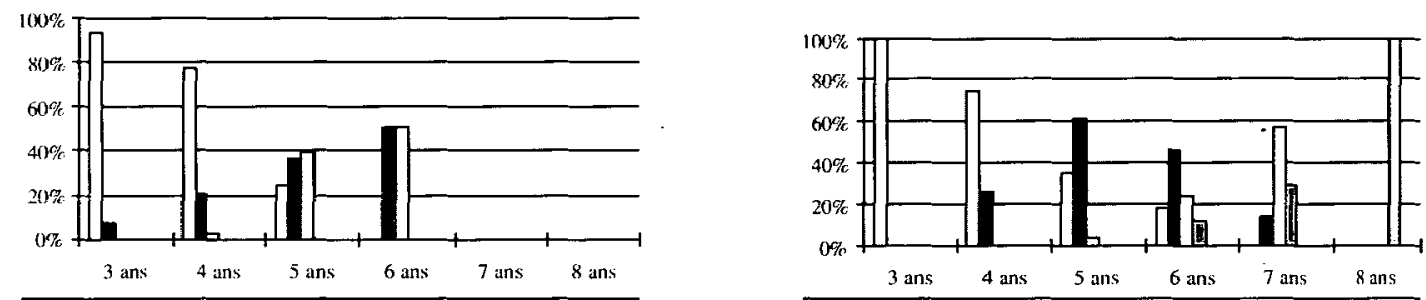

$\square \%$ dindividus à $n=0$ \% d'individus à $n=10 \%$ d'individus à $n=2$

$\square \%$ d'individus à $n=0$ Q $\%$ d'individus à $n=1 \quad 0 \%$ d'indjvidus à $n=2$ $\square \%$ dindividus à $\mathrm{n}=3 \square \%$ d'individus à $\mathrm{n}=4$

Figure 6

Répartition (\%) par groupe d'âge des aloses de première remontée (n $=0$ ) et de plusieurs reproductions ( $n=1$ à $n=5$ ) échantillonnées sur le Rhône en 1994, 1995 et 1996.

Figure 6

Repartition (\%) per age range of shads caught in the River Rhone in 1994, 1995 and 1996 (n = 0 : one upstream migration fishes, $n=1$ to $n=5$ : multispawned fishes).

Tableau VI

Nombre d'individus s'étant déjà reproduits une fois (1R) et plusieurs fois $(\geq 2 R)$ en fonction du sexe pour l'ensemble de la population d'aloses échantillonnées sur le Rhône en 1994, 1995 et 1996 (\% calculé par rapport au nombre total d'individus par sexe).

Table VI

Number of individuals which have already spawned one time (1R) or twice and more $(\geq 2 R)$ according to the entire population of shads caught in the River Rhone in 1994, 1995 and 1996.

\begin{tabular}{|c|c|c|c|c|c|c|c|c|c|c|}
\hline & \multicolumn{2}{|c|}{$1 \mathrm{R}$} & \multicolumn{2}{|c|}{$2 \mathrm{R}$} & \multicolumn{2}{|c|}{$3 R$} & \multicolumn{2}{|c|}{$4 R$} & \multicolumn{2}{|c|}{$5 R$} \\
\hline & $\mathrm{N}$ & $\%$ & $\mathrm{~N}$ & $\%$ & $\mathrm{~N}$ & $\%$ & $\mathbf{N}$ & $\%$ & $\mathrm{~N}$ & $\%$ \\
\hline mâles & 61 & 20,1 & 42 & 13,9 & 5 & 1,6 & 1 & 0,3 & 0 & 0 \\
\hline femelles & 131 & 29,7 & 84 & 19 & 33 & 7,5 & 4 & 0,9 & 1 & 0,2 \\
\hline
\end{tabular}


Le nombre de marques de reproduction observées sur les écailles varie de 1 à 5 . Cependant, $87,8 \%$ de la population totale de géniteurs échantillonnés sur les trois années et s'étant déjà reproduits présentent 1 à 2 marques de fraie sur leurs écailles (Tableau VI ; Fig. 7).

Le nombre de reproductions successives diminue avec l'âge du poisson. Par ailleurs, ce nombre est proportionnellement plus important chez les femelles que chez les mâles puisque $1,6 \%$ d'entre eux présente au moins 3 marques de fraie sur leurs écailles contre $7,5 \%$ chez les femelles (Tableau VI).

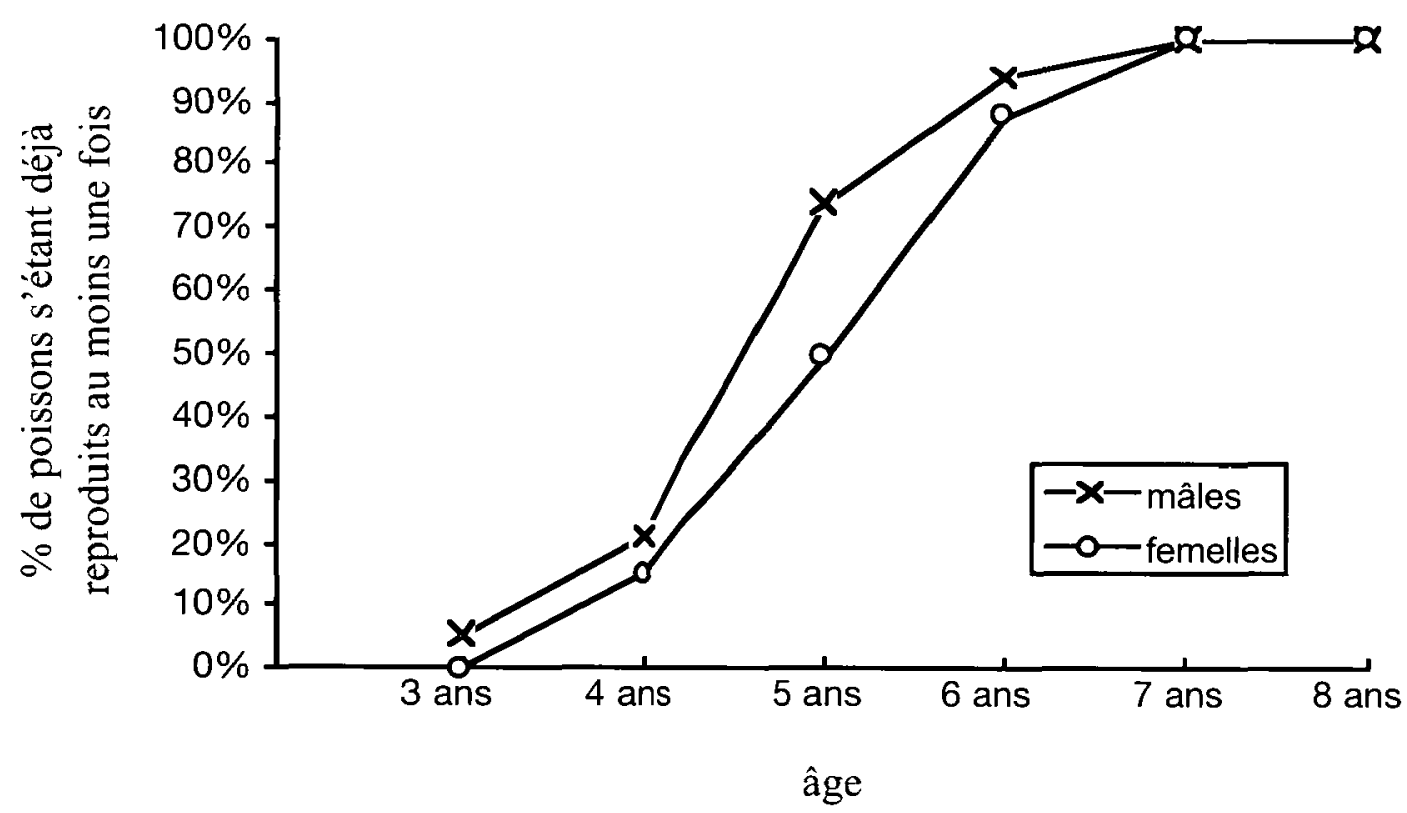

Figure 7

Evolution de la proportion (\%) de poissons s'étant déjà reproduits au moins une fois en fonction de l'âge chez les aloses échantillonnées sur le Rhône en 1994, 1995 et 1996.

\section{Figure 7}

Evolution of the proportion (\%) of fishes which have already spawned once or more according to age of shads sampled in the River Rhone in 1994, 1995 and 1996.

L'évolution du nombre de marques de reproduction en fonction de l'âge total du poisson montre qu'à partir de 6 ans, 90 à $100 \%$ des individus sont des poissons d'une ou plusieurs remontées (Fig. 6 et 7).

\section{DISCUSSION}

\section{Identification du taxon capturé}

Les mesures méristiques et morphométriques effectuées ont des valeurs moyennes tout à fait semblables à celles du principal taxon identifié par DOUCHEMENT (1981) comme celui d'Alosa fallax rhodanensis (Tableau II). II en est de même pour l'équation de la relation entre le nombre de branchiospines et la longueur totale du poisson. La valeur basse du coefficient de corrélation confirme, par ailleurs, la faible dépendance de ces deux paramètres chez le genre Alosa (DOUCHEMENT, 1981 ; MENNESSON-BOISNEAU et BOISNEAU, 1990 ; SABATIE, 1993). 
Cependant, la présente étude n'a pas permis de détecter ni les deux autres taxons identifiés par DOUCHEMENT (1981) sur le Rhône (grande Alose -Alosa alosa- et hybride), ni la grande Alose mentionnée par ANONYME (1975) et RAMEYE et al. (1976). Ceci peut être dû à la localisation dans le temps de l'échantillonnage des mesures morphométriques. En effet, ces mesures n'ont été effectuées qu'en fin de migration en 1995 et 1996 (à partir de début juin) alors que, sur les bassins où elles cohabitent, la grande Alose migre généralement plus tôt que l'Alose feinte (MENNESSON-BOISNEAU et BOISNEAU, 1990 ; SABATIE, 1993). Cependant, l'absence des deux autres taxons peut signifier également qu'ils ne sont plus présents dans la population d'aloses du Rhône et qu'alors, les caractéristiques biologiques analysées sur les trois années concernent essentiellement, voire totalement, le taxon d'Alose feinte du Rhône. Une telie situation semble se confirmer pour trois raisons : (1) la gamme de taille des poissons pour lesquels le comptage méristique a été fait couvre celle de la population totale étudiée, avec même un léger sur-échantillonnage pour ceux de grande taille qui pouvaient correspondre plus au taxon de la grande Alose possédant un gabarit moyen bien supérieur à celui de l'Alose feinte (MENNESSON-BOISNEAU et BOISNEAU, 1990; SABATIE, 1993); (2) au cours de ces quatre années d'études, DOUCHEMENT (1981) n'a trouvé qu'une proportion relativement faible d'hybrides $(15 \%)$ et de grandes aloses $(7,4 \%)$; ces poissons (adultes et immatures) présentaient un spectre de taille identique à celui de l'Alose feinte et ont été capturés tout au long de la migration aux mêmes périodes ; (3) le contrôle régulier des individus capturés par les pêcheurs professionnels durant toute la période de migration n'a pas révélé la présence d'individus autres que ceux appartenant au taxon Alosa fallax rhodanensis.

Par ailleurs, il reste à savoir si les individus, capturés par DOUCHEMENT (1981) et possédant en moyenne 70 et 50 branchiospines, pouvaient être réellement identifiés. comme étant respectivement des grandes aloses et des hybrides entre cette dernière et l'Alose feinte du Rhône.

\section{Caractéristiques des adultes}

\section{Les résultats font ressortir plusieurs points :}

* La confirmation de deux paramètres démographiques caractéristiques des adultes du genre Alosa, à savoir une maturité sexuelle plus tardive des femelles et une croissance plus élevée de ces dernières à âge égal comparées à celles des mâles (APRAHAMIAN, 1981; CASSOU-LEINS et CASSOU-LEINS, 1981 ; DOUCHEMENT, 1981 ; MENNESSON-BOISNEAU et BOISNEAU, 1990 ; SABATIE, 1993).

* L'existence de variations annuelles naturelles plus ou moins fortes selon le paramètre démographique considéré, comme cela a déjà été montré sur les taxons atlantiques (MENNESSON-BOISNEAU et BOISNEAU, 1990 ; TAVERNY, 1991; SABATIE, 1993). Ainsi, la taille moyenne annuelle par classe d'âge évolue en particulier pour les mâles. Cependant, la structure d'âge et le rapport des sexes montrent une variabilité qui semble pouvoir s'expliquer en grande partie par des différences inter-annuelles de techniques d'échantillonnage. Il en est de même de la taille moyenne en fonction de l'âge. En effet, en 1996, la large gamme de taille de mailles des filets utilisés a permis d'échantillonner correctement la population de mâles, alors que les deux autres années, les engins employés ont favorisé la capture des mâles les plus grands et les plus âgés. Cette variabilité annuelle de la structure d'âge en fonction des techniques et des sites d'échantillonnage apparaît plus marquée pour les mâles que pour les femelles qui ont, quelle que soit l'année, un gabarit plus important. De même, la variation observée sur le rapport des sexes au cours des trois années peut être expliquée par les différences dans les sites de prélèvement. Ainsi, en 1994, la majorité des captures a été effectuée en amont du barrage alors que les années suivantes, elle a été réalisée essentiellement en aval. Or, il semble que les femelles, compte tenu de leur embonpoint et de leur taille, soient en.règle générale plus gênées dans leur migration en amont par la présence d'un obstacle que les mâles (MENNESSON-BOISNEAU et BOISNEAU, 1990). Par ailleurs, la principale frayère existant actuellement sur le Rhône est située en aval du barrage de Vallabrègues. La conjugaison de ces deux éléments pourrait alors entraîner une plus forte 
concentration de femelles, et donc de poissons de grande taille (croissance plus forte des femelles) en aval du barrage, et ainsi expliquer d'une part la taille et l'âge moyens plus élevés et d'autre part la plus grande proportion de femelles capturées en 1995. Par ailleurs, le rapport des sexes équilibré observé en 1996 (et identique à la valeur de 1994) est dû à une modification voulue au cours du temps des techniques d'échantillonnage afin d'obtenir un nombre identique de mâles et de femelles pour les études taxonomiques.

La comparaison des résultats obtenus sur les trois campagnes avec ceux présentés par DOUCHEMENT (1981) montre essentiellement des différences dans la taille moyenne par classe d'âge et la valeur du rapport des sexes de la population de géniteurs. En effet, à gamme de taille égale, les aloses adultes analysées par ce dernier auteur présentent une structure d'âge assez proche même s'il note un spectre d'âge des mâles plus faible (de 3 à 5 ans) que celui noté dans cette étude (de 3 à 8 ans). En revanche, les tailles moyennes par classe d'âge sont plus élevées que celles citées par DOUCHEMENT (1981), quel que soit le sexe. II faut cependant signaler que les résultats obtenus par ce dernier auteur sont cumulés sur quatre ans. Néanmoins, les écarts de taille constatés entre les deux périodes (1975-1978 et 1994-1996) pourraient s'expliquer a priori plus par une modification du taux de croissance de la population de géniteurs que par des différences d'ordre méthodologique. Ces dernières pourraient provenir moins des techniques de capture utilisées que d'une interprétation différente des écailles. A cet égard, il faut souligner que, s'il est facile de détecter correctement les anneaux de pontes, la localisation des autres marques d'arrêt de croissance reste toujours plus délicate et notamment celles des premier et dernier hivers.

De même, DOUCHEMENT (1981) observe un rapport des sexes très défavorable aux femelles $(2,1$ mâles pour une femelle), soit plus de deux et huit fois la valeur observée respectivement en 1994 et 1995. Le nombre plus élevé de mâles dans la population migrante a souvent été noté dans le cas de la grande Alose (MENNESSON-BOISNEAU et BOISNEAU, 1990 ; SABATIE, 1993). Cette différence de rapport des sexes notée entre les deux études peut provenir en partie du niveau de sélectivité des types d'engins de pêche utilisés pour capturer des poissons et expliquer la plus faible taille moyenne de la population échantillonnée par DOUCHEMENT (1981). En effet, les carrelets et les globes employés par ce dernier sont des engins non sélectifs comparés aux filets à grande maille qui paraissent avoir sélectionné des individus de plus grande taille (donc des femelles) lors des campagnes 1994 et 1995. Néanmoins, il reste difficile de connaître, compte tenu des données disponibles, la part respective prise par les facteurs d'origine naturelle ou liés aux méthodes d'échantillonnage dans les fluctuations du rapport des sexes constatées entre les deux périodes. Ceci semble une règle générale pour toutes les populations d'aloses qui ont été étudiées (MENNESSONBOISNEAU, APRAHAMIAN, SABATIE et CASSOU-LEINS, données non publiées).

* La présence d'un phénomène important d'itéroparité (jusqu'à six remontées) caractéristique de l'Alose feinte dans toute son aire de répartition atlantique (APRAHAMIAN, 1981; MENNESSON-BOISNEAU et BOISNEAU, 1990; TAVERNY, 1991; ALEXANDRINO, 1990), à l'exception des populations marocaines (SABATIE, 1993). Cependant, la proportion des individus s'étant reproduits au moins une fois dans les populations échantillonnées en 1994, 1995 et 1996 est en moyenne le double de celle observée par DOUCHEMENT (1981) à la fin des années 70 . Cette augmentation ne semble pas s'expliquer par des différences d'ordre méthodologique puisque la structure d'âge des géniteurs reste assez proche entre les deux périodes et que les marques de reproduction sont très facilement identifiables sur les écailles. II semble donc que ce phénomène d'itéroparité se soit accentué naturellement puisque la proportion d'individus s'étant reproduits au moins une fois et au moins deux fois dans la population étudiée est très supérieure (respectivement $36 \%$ et $14 \%$ pour les mâles et $57 \%$ et $19 \%$ pour les femelles) à celle observée par DOUCHEMENT (1981) (respectivement $21,1 \%$ et $2,1 \%$ pour les mâles et $14,5 \%$ et $10,8 \%$ pour les femelles). Ceci laisse supposer une augmentation du taux de survie générale de la population d'adultes, et plus particulièrement de celle des femelles après la reproduction. Enfin, la proportion d'individus itéropares trouvée 
actuellement dans la population d'Alose feinte du Rhône la rapproche fortement de celle de la Loire (MENNESSON-BOISNEAU et BOISNEAU, 1990), de la Garonne (TAVERNY, 1991) et du Tage (ALEXANDRINO, 1990).

* L'existence possible de deux spécificités biologiques du taxon rhodanien, déjà mises en évidence par DOUCHEMENT (1981) :

- Une maturité sexuelle légèrement plus tardive, quel que soit le sexe, par rapport à celle des populations d'Alose feinte atlantique de nos côtes (MENNESSON-BOISNEAU et BOISNEAU, 1990 ; TAVERNY, 1991). Ainsi, la majorité des mâles et des femelles se reproduit respectivement à 3-4 ans et 4-5 ans. Cependant, au vu des résultats obtenus, il semble que le décalage de l'âge moyen de maturité sexuelle se soit réduit entre les populations rhodanienne et atlantique. En effet, actuellement, une proportion plus élevée de mâles et de femelles se reproduirait respectivement à l'âge de 3 ans $(38,3 \%)$ et 4 ans $(54,2 \%)$, comparée à celle notée par DOUCHEMENT (1981) à la fin des années 1970 (respectivement $21,6 \%$ et 38,7 \%). Ceci pourrait être relié au taux de croissance de la population et confirmerait alors l'augmentation de ce dernier, évoqué précédemment, entre les deux périodes.

- Une plus grande longévité, comparée à celle de toutes les populations d'Alose feinte atlantique. Cette caractéristique de l'Alose du Rhône est en étroite liaison avec le fort taux d'itéroparité et la maturité plus tardive de la population. Ainsi, le spectre de classe d'âge chez les géniteurs est plus large que celui de l'Alose feinte atlantique ( 3 à 6 ans), à l'exception des populations de la Severn et de la Wye (Pays de Galles) (2 à 7 ans : APRAHAMIAN, 1985), et semblable à celui de la grande Alose ( 3 à 8 ans) (MENNESSON-BOISNEAU et BOISNEAU, 1990 ; TAVERNY, 1991 ; SABATIE, 1993). De fait, l'AMR de l'Alose du Rhône est toujours plus élevé que celui du taxon atlantique; il reste assez proche (principalement pour les femelles) de celui de la grande Alose des côtes françaises (MENNESSON-BOISNEAU et BOISNEAU, 1990 ; TAVERNY, 1991), mais est plus faible que l'AMR de cette espèce présente dans les cours d'eau marocains et portugais (SABATIE et al., 1996).

\section{CONCLUSION}

L'analyse des données récoltées sur la population d'aloses du Rhône au cours des trois premières campagnes apporte un certain nombre de résultats intéressants et permet d'établir des hypothèses si on la compare au travail réalisé par DOUCHEMENT (1981) à la fin des années 1970 :

* La présence dans la population actuellement échantillonnée d'un seul taxon Alosa fallax rhodanensis, identifié jusqu'à présent comme une sous-espèce de l'Alose feinte. Ses critères morphologiques (notamment le faible nombre de branchiospines), certaines de ses caractéristiques démographiques (itéroparité) ainsi que ses périodes de migration et de reproduction (BAGLINIĖRE et al., 1996) le rattachent sans contestation possible à l'espèce A. fallax. En revanche, d'autres caractéristiques biométriques et démographiques (gabarit moyen élevé, forte longévité) le rattacheraient plus à la grande Alose.

* L'existence de possibles modifications de la stratégie démographique de la population entre les deux périodes, en raison : (1) d'une augmentation du taux de croissance qui a pu entraîner une diminution de l'âge de première maturité sexuelle, permettant ainsi un renouvellement plus rapide du stock, (2) d'un niveau d'itéroparité plus élevé par suite d'une meilleure survie des géniteurs après la fraie (effort migratoire devenu plus réduit), plus spécialement chez les femelles, permettant alors une augmentation du potentiel reproducteur de la population actuelle.

Cependant, ces premiers résultats restent partiels et ces hypothèses doivent être prises avec précaution, suite : (1) à l'absence d'homogénéité dans les techniques de capture utilisées et à la variabilité des sites d'échantillonnage, (2) à l'interprétation délicate des écailles dans le cas de poissons très âgés ou présentant un phénomène d'érosion important du bord de l'écaille. 
Ainsi. lors de la prochaine campagne. il sera nécessaire (1) d'augmenter l'effort d'échantillonnage à la fois dans le temps, en utilisant des techniques de capture identiques d'une année a l'autre, et dans l'espace. en effectuant des captures sur des sites fixes situes en aval du barrage et non perturbés par la présence de cet obstacle (utilisation de la pêche professionnelle et amateur aux engins qui reste encore assez importante sur le Rhóne (CHANGEUX, 1994)). (2) de multiplier le nombre de mesures morphologiques, de prélèvements d'écailles et d'autres structures osseuses, (3) d'établił une clé taille-âge fiable en prenant en compte les populations de juveniles et dimmatures et en complétant les observations scalimetriques par celles des otolithes notamment. (4) de caracteriser génétiquement la population par l'analyse électrophorétique des protéines.

\section{REMERCIEMENTS}

Cette ètude a été réalisée dans le cadre d'une convention d'étude passée avec la DIREN Rhône-Alpes. Elle fait partie d'un programme scientifique sur les aloses du Rhòne, etabli en collaboration avec le Laboratoire d'Ecologie Aquatique INRA de Rennes. Ie Laboratoire d'Halieutique ENSA de Rennes. 'Association Migrateurs Rhône-Méditerranée et le Laboratoire d'Ecologie des Systemes Fluviaux CNRS d'Arles. Nous adressons nos plus sincères remerciements a Yann MAZENS pour son aide précieuse lors de la campagne de terrain 1996.

\section{BIBLIOGRAPHIE}

ALEXANDRINO P., 1990. Primeiros dados sobre a eficacia da eclusa Borland da barragem de Belver, rio Tejo. Alguns aspectos sobre a biologia das especies migradoras. Actas da 2 Conferencia Nacional sobre a qualidade do ambiente, 2, 1-15.

ALEXANDRINO P., 1996. Estudo de populaçoes de savel (Alosa alosa L.) e savelha (Alosa fallax Lacépède). Analise da diferenciaçao interspecifica, subestructuraçao e hibridaçao. Thèse Doctorat. Université de Porto, $180 \mathrm{p}$

ALEXANDRINO P., FERRAND N., ROCHA J., 1996. Genetic polymorphism of a hemoglobin (HB) chain and adenosine deaminase (ADA; E. C. 3. 5. 4. 4) in European shads. Alosa spp. : evidence for the existence of two distinct genetic entities with natural hybridization. J. Fish. Biol., 48, 447-456.

ANONYME, 1975. Les obstacles à la migration des poissons du Rhône dans le département du Gard. Rapport Division Qualité des Eaux, Pêche et Pisciculture, C.T.G.R.E.F., 54 p.

APRAHAMIAN M.W., 1981. Aspects of the biology of the twaite Shad (Alosa fallax) in the rivers Severn and Wye. Proceedings of the 2 nd British Freshwater Fisheries Conference, 111-119.

APRAHAMIAN M.W. 1985. The effect of migration of Alosa fallax fallax (Lacépède) into freshwater, on branchial and gut parasites. J. Fish. Biol., 27, 521-532.

BAGLINIERE J.L., SABATIE M.R., lE CORRE M., MENELLA J.Y., PONT D., 1996. Premiers éléments de caractérisation biologique et taxonomique de l'Alose feinte du Rhône (1994 et 1995). Rapport contrat INRA-DIREN Rhōne-Alpes, 23 p.

BIANCO P.G., 1995. Mediterranean endemic freshwater fishes of Italy. Biol. Conserv., 72 , $159-170$.

BLANCHARD E., 1866. Les poissons deau douce de la France. BAILLERE et fils (Ed.). Paris, $656 \mathrm{p}$.

BRIOCCHI A.. 1896. La pisciculture dans les eaux douces. Bibliothèue des Sciences et de I'Industrie, Paris, $325 \mathrm{p}$.

CASSOU-LEINS F., CASSOU-LEINS J.J., 1981. Recherches sur la biologie et l'halieutique des migrateurs de la Garonne et principalement de l'Alose. Thèse 3ème cycle. Institut National Polytechnique de Toulouse, $393 \mathrm{p}$. 
CHANGEUX T.. 1994. Structure des peuplements de poissons à l'echelle du bassin rhodanien. These Doctorat. Universite Claude-Bernard Lyon I. $241 \mathrm{p}$

DOUCHEMENT C.. 1981. Les aloses des fleuves français. Alosa fallax Lacépède, 1803 et Alosa alosa Linné. 1758. Biométrie. Ecobiologie. Autonomie des populations. These 3" cycle. Université des Sciences et Techniques du Languedoc. Montpellier. $377 \mathrm{p}$.

FAHY E., 1978. Variation in some biological characteristics of British sea trout. Salmo trutta. in Ireland. J. Fish. Biol.. 13. 123-138.

GALLOIS C., 1946. L'Alose du Rhône. Bull. Fr. Piscic.. 141, 162-176.

GALLOIS C.. 1947. L'Alose du Rhône. Bull. Fr. Piscic.. 144. 130-136.

HOESTLANDT H., 1958. Reproduction de l'Alose atlantique (Alosa alosa Linne) et transfert au bassin mediterraneen. Verhandlungen. Internationale Vereineigung für theorische und angewandte Limnologie. 13. 736-742.

LARINIER M.. RIVIER B., ALLARDI J.. TROCHERIE F., 1978. Possibilites de franchissement du seuil de Beaucaire par les aloses du Rhône. Bull. Fr. Piscic., 268, 107-120.

LEGAULT A., 1993. Expertise pour l'accès des aloses et des anguilles aux affluents du Rhône. Inventaires des sites potentiels de frai daloses. Rapport Fish Pass. Association Migrateurs Rhône-Méditerranée. $97 \mathrm{p}$.

MENNESSON-BOISNEAU C., BOISNEAU P., 1990. Recherches sur les aloses du bassin de la Loire: Migration. Répartition. Reproduction. Caractéristiques biologiques et Taxonomie des aloses (Alosa sp.). Thèse Doctorat, document de synthèse. Universités de Rennes et de Paris Val de Marne, $143 \mathrm{p}$.

MENNESSON-BOISNEAU C., BAGLINIĖRE J.L., 1992. Mise au point d'une méthode de determination de lâge de la grande Alose (Alosa alosa). In Tissus durs et âge individuel des vertébrés, BAGLINIERE J.L., CASTANET J.. CONAND F. et MEUNIER F. (Eds), ORSTOM-INRA, Paris. 221-231.

QUIGNARD J.P., KARTAS $F$, 1977. Les aloses feintes, Alosa fallax (Lacépède, 1803), poissons clupéiformes de l'Atlantique nord-est et de la Méditerranée: Etude des caractères numériques. Bull. MNHN. 3ème serie. 501, 1241-1256.

QUIGNARD J.P., DOUCHEMENT C., 1991. Alosa fallax modanensis (Roule, 1924). In The freshwater fishes of Europe. Clupeidae, Anguillidae. HOESTLANDT $H$. (Ed.), Aula-Verlag Wiesbaden, 274-289.

RAMEYE L. KIENER A., SPILLMANN C.P., BIOUSSE J., 1976. Aspect de la biologie de l'alose du Rhône ; Pêche et difficultés croissantes de ses migrations. Bull. Fr. Piscic. 263, 50-76.

ROULE L., 1924. Les aloses des eaux douces de France. Bull. Soc. Zool. France. 49. 265-266.

ROULE L., 1925. Les poissons des eaux douces de France. Presses Universitaires de France (Ed.). Paris, $228 p$

SABATIE M.R., 1993. Recherches sur lEcologie et la Biologie des aloses au Maroc (Alosa alosa Linné. 1758 et Alosa fallax Lacépède. 1803) : Exploitation et Taxonomie des populations atlantiques. Bioécologie des aloses de l'Oued Sebou. Thèse Doctorat, Université de Bretagne Occidentale, Brest. $326 \mathrm{p}$.

SABATIE M.R., ALEXANDRINO P., BAGLINIERE J.L., 1996. Comparaison des caractéristiques biologiques de l'Alose vraie (Alosa alosa Linne, 1758) dans l'Oued Sebou (façade nord-atlantique du Maroc) et dans le fleuve Lima. Cybium. 20 (3). 59.73.

TAVERNY C., 1991. Contribution à la connaissance de la dynamique de populations daloses (Alosa alosa et Alosa fallax) dans le systeme fluvio-estuarien de la Gironde: Pêche. Biologie, Ecologie. Thèse Université de Bordeaux I. $451 \mathrm{p}$.

WHITEHEAD P.J.P. 1985. FAO species catalogue. 7 : Clupeoid fishes of the world (Suborder Clupeoidei). An annoted and illustrated catalogue of the Herrings, Sardines, Pilchards, Sprats, Anchovies and Wolf-Herrings. Part 1 : Chirocentridae. Clupeidae and Pristigasteridae. FAO Fisheries Synopsis. 125. 7. Part 1. 303 p. 